\title{
Active control of drag noise from a small axial flow fan
}

\author{
Jian Wang and Lixi Huang ${ }^{\text {a) }}$ \\ Department of Mechanical Engineering, The Hong Kong Polytechnic University, Kowloon, Hong Kong
}

(Received 10 November 2005; revised 12 April 2006; accepted 20 April 2006)

\begin{abstract}
Noise sources in an axial flow fan can be divided into fluctuating axial thrust forces and circumferential drag forces. For the popular design of a seven-blade rotor driven by a motor supported by four struts, drag noise dominates. This study aims to suppress the drag noise globally by active control schemes. Drag noise features a rotating dipole and it has to be cancelled by a secondary source of the same nature. This is achieved experimentally by a pair of loudspeakers positioned at right angles to each other on the fan rotational plane. An adaptive LMS feedforward scheme is used to produce the control signal for one loudspeaker and the time derivative of this signal is used to drive the other loudspeaker. The antisounds radiated by the two loudspeakers have a fixed phase relation of $90^{\circ}$ forming a rotating dipole. An open-loop control scheme is also implemented for the purpose of comparison and easier implementation in real-life applications. The results show that the globally integrated sound power is reduced by about $13 \mathrm{~dB}$ for both closedand open-loop schemes. A possible limiting factor for the cancellation performance is found to be the presence of higher order modes of drag noise. (C) 2006 Acoustical Society of America.
\end{abstract}

[DOI: 10.1121/1.2204443]

PACS number(s): 43.50.Ki [SFW] Pages: 192-203

\section{INTRODUCTION}

Small axial flow fans make noise much like large compressors and turbines, but the small number of rotor blades renders easier physical interpretation of the source characteristics (Huang, 2003). A brief review of the noise sources and noise abatement techniques specific to small axial flow fans is given in a previous study (Wang et al., 2005). Unsteady forces acting on the blades are the origin of most fan noise. The forces acting on a blade can be divided into a thrust component along the rotating axis, and a drag component in the circumferential direction of the rotor. The final radiated noise is a result of complex acoustic interference between these two force components on each blade, and of sounds from all blades. The previous study deals with the active control of noise radiated by the thrust forces arising from the rotor-strut interactions. This study extends the control to drag noise, which is a far more complex component. In what follows, a brief summary is given for the existing active fan noise control with emphasis on the issue of directivity pattern, followed by the description of the configurations used in the current study.

Active fan noise control is further divided into active minimization of the source strength by interfering with the aerodynamics (e.g., Neuhaus et al., 2003; Rao et al., 2001; Simonich et al., 1993), and the cancellation of the radiated sound by secondary sources (e.g., Gerhold, 1997; Thomas et al., 1993, 1994; Quinlan, 1992; Lauchle et al., 1997; Gee and Sommerfeldt, 2004). One common feature in most reported works is that the rotational plane of the fan is placed in a baffle in order to simplify the acoustic field before the global control is contemplated. The effect of such a baffle on

\footnotetext{
${ }^{\text {a) }}$ Author to whom correspondence should be addressed. Electronic mail: mmlhuang@polyu.edu.hk
}

the acoustic directivity has not been quantified. However, it may be speculated that the effects on different components of the noise sources are different. As a result, the acoustic interference between these components would also change, leading to a changed directivity pattern. Recently, Gerard et al. (2005) tried to use a single loudspeaker to cancel the tonal noise of an axial flow fan without using a baffle. They assume that the fan noise source can be represented by a single dipole source, but their measured acoustic directivity shows a tilted pattern and, not surprisingly, only part of the sound field can be cancelled. In fact, the tilted directivity pattern is a result of acoustic interference between waves radiated by the axial thrust component and the circumferential drag component. A detailed analysis of such interference has been given by Huang and Wang (2005) based on experimental data.

The acoustic radiation efficiency depends strongly on the difference between the spatial index of spinning pressure modes and the frequency index of the radiated sound (Tyler and Sofrin, 1962; Lowson, 1970), $\nu=m B-k S$, where $B$ and $S$ are the numbers of rotor blades and stationary struts, respectively, $m$ is the harmonic index and $k$ is any integer. Careless designs of a cooling fan often create a rotor-strut interaction in which the effective number of strut is $S=1$, for which the thrust noise radiates at the leading mode of $\nu=0$ for $k=m B$. The leading mode thrust noise has a simple directivity pattern and a previous study has demonstrated the effectiveness of active control by using a single loudspeaker (Wang et al., 2005). A more careful design of a cooling fan, however, features more drag noise than thrust noise (Huang and Wang, 2005). Drag noise is radiated by rotating dipoles and its leading order mode features $\nu= \pm 1$. It is far more complex acoustically than the thrust noise and, to the best of the authors' knowledge, there has not been a three-dimensional measurement of a fan noise radiation in which such a leading mode 


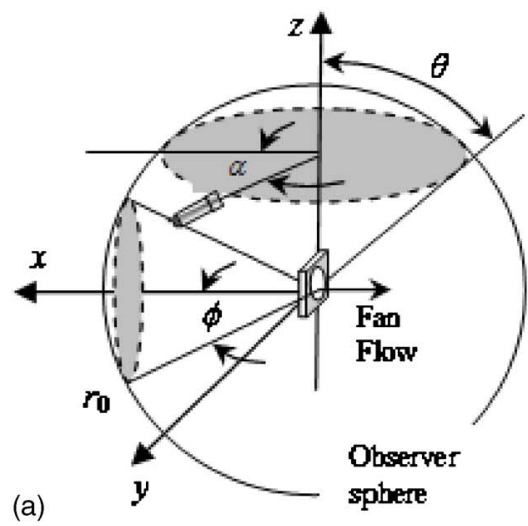

(b)

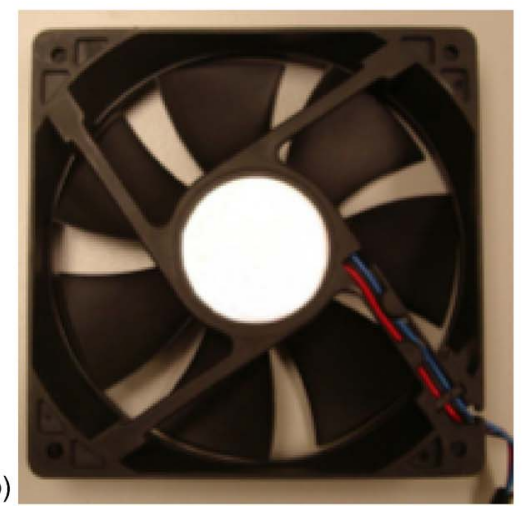

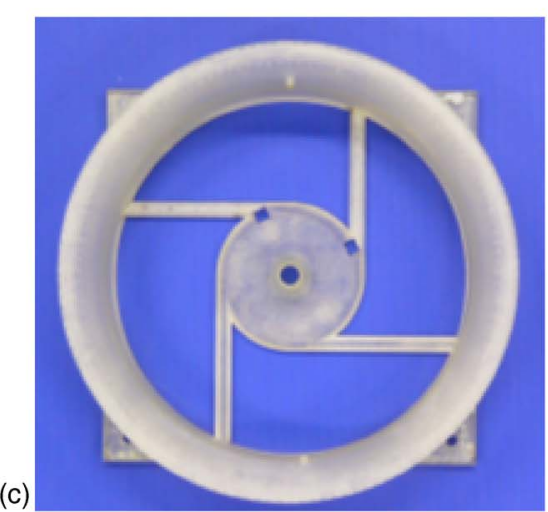

FIG. 1. (Color online) (a) Coordinate system used in theory and experiments. (b) The back view of the sample fan. (c) The front view of the modified fan casing.

dominates. As is shown in later sections, the leading mode drag noise can be approximated by two stationary dipoles operating in a fixed phase relation. This being true, two loudspeakers can be used to construct the antisound for the drag noise. This study aims to find out, experimentally, whether such antisound works and to what extent it may globally suppress the drag noise. The specific objectives of this study are the following: $(i)$ to measure the percentage of sound power from a well-designed fan that can be attributed to the leading mode drag noise; (ii) to study, by numerical simulation, the extent to which the sound radiated by a pair of loudspeakers can globally cancel the drag noise from the interaction of seven rotor blades and four struts; (iii) using the filtered-X least-mean-square algorithm, conduct the active control for the drag noise with a set of optimal parameters found by numerical simulation; (iv) compare the performance of the closed-loop control with an open-loop control; and $(v)$ analyze the results and determine the crucial factor that controls the overall performance.

In what follows in Sec. II, the characteristics of a real sample fan and the design improvement are described briefly to demonstrate how a well-designed fan features mainly the drag noise. In Sec. III, numerical simulation is conducted to predict the parametric influence of various factors present in a real control rig, such as the location of the error microphone and the distance between the fan center and the secondary sources. Section IV describes the experimental results of both closed-loop and open-loop controls. Analysis of the residual noise is also described, leading to conclusions in Sec. V.

\section{ACOUSTICS OF THE SAMPLE FAN}

The coordinate system is defined in Fig. 1(a). The axialflow fan is shown standing vertically up along the $+z$ axis, and the sound radiated by the fan is surveyed by a microphone over a sphere of radius $r_{0}$ from the fan center. When viewed from upstream, the fan rotates counter-clockwise. The observer sphere is described by a latitudinal angle $\theta$ $\in[0, \pi]$ and a longitudinal angle $\alpha \in[0,2 \pi]$. An alternative latitudinal angle is $\phi$ measured from the $+x$ axis. Note that $\phi$ overlaps with $\alpha$ when $\phi$ is measured on the central horizontal plane of $\theta=90^{\circ}$, or $z=0$, but they are, strictly speaking, different. Note that $\phi$ is used in theoretical derivations while $\alpha$ is used for directivity measurements and discussions on the central horizontal plane of $z=0$. To measure the effectiveness of the global control, three directivity measurement planes are used: $\theta=30^{\circ}, 60^{\circ}, 90^{\circ}$, in addition to the top point at $\theta$ $=0^{\circ}$.

\section{A. Theory}

In order to analyze the noise made by the sample fan, the basic theory of the rotor-strut interaction acoustics is summarized below. The tonal sound radiated by the unsteady force on the rotor blades arising from the interaction of $B$ blades with $S$ struts of equal size and uniform spacing is given by (Lowson 1965, Lowson 1970).

$$
\begin{aligned}
c_{m B}^{(\text {rotor })}= & \frac{i m \omega B^{2} S}{2 \pi c_{0} r_{0}} \sum_{k=-\infty}^{+\infty} i^{-\nu}\left(T_{k S}^{(\text {rotor })} \cos \phi-\frac{\nu}{m B M} D_{k S}^{(\text {rotor })}\right) \\
& \times J_{\nu}(m B \mathrm{M} \sin \phi), \quad \nu=m B-k S
\end{aligned}
$$

where $c_{m B}^{\text {(rotor) }}$ is the complex pressure amplitude at the frequency of $m B \times \mathrm{rps}$, rps is the rotations per second for the rotor, $\omega=2 \pi(\mathrm{rps}), k S$ is the frequency index in the spectrum of the unsteady force components of thrust $T$ and $\operatorname{drag} D$ on the rotor blades, $c_{0}$ is the speed of sound, $\mathrm{M}$ is the Mach number defined as $\omega r_{s} / c_{0}, r_{s}$ is the radius at which the interaction occurs, and $k$ is any integer. The frequency index differential, $\nu=m B-k S$, or the index of the spinning pressure mode (Tyler and Sofrin, 1962), is the most important parameter. The noise radiated by the corresponding interaction forces on the struts is found when the source terms of $T_{k S}^{\text {(rotor) }}, D_{k S}^{\text {(rotor) }}$ are replaced by $T_{m B}^{\text {(strut) }}, D_{m B}^{\text {(strut) }}$ in which the frequency index $k S$ is replaced by $m B$ as each strut experiences the interaction events with $B$ rotor blades per rotational cycle.

The two most effective modes of sound radiation are explained physically by Huang (2003). When $m B=k S$, the thrust forces exerted by all blades occur simultaneously and simply add up, and the noise radiated is a simple dipole whose axis is along the rotational axis. However, the situation for the drag force is different. Drag force changes direction once per cycle, so the frequency perceived by a stationary observer is $k S \pm 1$ and no noise is radiated at $m B=k S$, as 
can be seen by the numerator $\nu$, which vanishes, in the drag noise term in Eq. (1). For this reason the leading drag noise radiation mode has $\nu= \pm 1$. Higher order modes radiate sound by way of the Doppler effect, which is small for typical computer cooling fan operating at a low Mach number below 0.1 . However, their presence alters the appearance of the acoustic directivity quite dramatically (Huang and Wang, 2005). The directivity patterns of the leading and the next higher order modes are summarized below:

$$
\begin{aligned}
& p_{T 0} \propto \cos \phi, \quad p_{T 1} \propto \sin \phi \cos \phi, \\
& p_{D 1} \propto \sin \phi, \quad p_{D 2} \propto \sin ^{2} \phi,
\end{aligned}
$$

where $p$ is the radiated sound pressure, subscripts $T$ and $D$ indicate the sources of thrust and drag forces, respectively, and the numerical subscripts 0,1 , and 2 indicate the value of $|\nu|$. The distinct directivity patterns make it possible to separate the four mechanisms by simultaneously measuring sound at four symmetrical angular positions of $\alpha_{1}, \alpha_{2}=\pi$ $-\alpha_{1}, \alpha_{3}=\pi+\alpha_{1}, \alpha_{4}=2 \pi-\alpha_{1}$, where $\alpha_{1} \in[0, \pi / 2]$ is the position of the first microphone on a horizontal measurement plane. The four noise components given in Eq. (2) may be extracted as follows (Huang and Wang, 2005):

$$
\begin{aligned}
& p_{T 0}=\frac{p_{1}-p_{2}-p_{3}+p_{4}}{4}, \quad p_{T 1}=\frac{p_{1}-p_{2}+p_{3}-p_{4}}{4}, \\
& p_{D 1}=\frac{p_{1}+p_{2}-p_{3}-p_{4}}{4}, \quad p_{D 2}=\frac{p_{1}+p_{2}+p_{3}+p_{4}}{4} .
\end{aligned}
$$

\section{B. Noise from the original sample fan}

The sample fan used in this study of active control is the improved version of a computer cooling fan taken from the market (Delta AFB1212SH series), which is shown in Fig. 1(b). The original fan has a casing diameter of $120 \mathrm{~mm}, B$ $=7$ rotor blades, and $S=4$ downstream struts. The design speed is $3000 \mathrm{rpm}$. Two aspects of this sample fan contribute to loud noise. The first is that the circular inlet flow passage is intercepted by the square frame distorting the inlet flow to become one with a four-lobe pattern. The effect is similar to a set of four inlet vanes. Vortices are generated by the four edges and they are not entirely coordinated with a phase locked to the rotation. As such, they should contribute to both discrete and broadband parts of the noise spectrum. The second feature is that the strut carrying the electrical wires is larger than the other three. The effect of the extra size of the large strut can be considered to be that of a single strut, $S$ $=1$, which is a very efficient noise source for both thrust and drag noise components when it interacts with a rotor of any blade number. Details of the noise mechanism of these two features are studied by Huang and Wang (2005) for a smaller cooling fan $90 \mathrm{~mm}$ in diameter. Correction of these two features led to a power reduction of about $10 \mathrm{~dB}$ in tonal noise for that small fan. The same procedure is followed here, and the new casing design is shown in Fig. 1(c). A full circular inlet bellmouth is installed and the four struts are made equal in size. It is emphasized that the active control technique is applied on the acoustically improved design shown in Fig. 1(c) instead of the original fan.

The acoustic directivity patterns for the original and improved fan are shown in Fig. 2. Figures 2(a)-2(c) are for the original fan shown in Fig. 1(b), while Figs. 2(d) and 2(e) are for the improved fan whose casing is shown in Fig. 1(c). Figure 2(a) shows the overall noise (outer dashed line), random noise (dash-dot line with a pattern almost parallel with the horizontal axis), rotary noise (thin solid line), and the BPF component of the rotary noise (thick solid line). Here, random noise is defined as the difference between the overall noise and the rotary noise, and the rotary noise is obtained by the synchronous average with the help of the tachometer signal. The air flow is drawn from the left, where $\alpha=0$ is labeled, to the right.

As shown in Fig. 2(a), the major axis of the overall noise pattern is tilted along the direction of $\alpha=30^{\circ}, 210^{\circ}$. This oblique distribution is a result of the interference between the drag noise, which spans out on the rotational plane, and the thrust noise, which beams along the rotational axis. The two components are separated in Fig. 2(b). The integrated power of the BPF component of the drag noise (thin line) is $S W L_{\mathrm{BPF}}^{(\mathrm{drag})}=50.4 \mathrm{~dB}$, which is $4.8 \mathrm{~dB}$ higher than the thrust noise (thick line). Such a prominent contribution from the thrust noise is caused by the extra size of the strut carrying the wires, which acts like a single strut, $S=1$. For the spectral component of $k=B$, it gives the leading mode thrust noise radiation with $\nu=m B-k S=0$. Meanwhile, the modes of $k$ $=B-1, B+1$ also give the leading mode drag noise radiation with $\nu= \pm 1$, which is superimposed on the radiation of the drag noise by

$$
\nu=m B-k S=1 \times 7-2 \times 4=-1
$$

caused by the interaction between the seven rotor blades with the four struts with $k=2$. The extracted drag noise pattern shown in Fig. 2(b) also features asymmetry with respect to the rotational axis. More noise is radiated towards $\alpha=270^{\circ}$ than $\alpha=90^{\circ}$. This is caused by the interference between the leading mode drag noise, $p_{D 1} \propto \sin \phi$, and the higher order drag noise, $p_{D 2} \propto \sin ^{2} \phi$, the latter being, presumably, originated from the factor of $S=1$. Using the method of noise source and modal decomposition of Eq. (3), the leading mode drag noise has the sound power of $S W L_{\mathrm{BPF}}^{(D 1)}=50.8 \mathrm{~dB}$ while the higher order drag noise has $S W L_{\mathrm{BPF}}^{(D 2)}=33.2 \mathrm{~dB}$. The typical sound pressure level (SPL re $20 \mu \mathrm{Pa}$ ) spectrum measured at the angle of $\alpha=230^{\circ}$ is shown in Fig. 2(c), which shows high peaks for the first few BPF harmonics. Note that the peaks on the harmonics of the BPF may be a little higher than the result from the raw data, and this is caused by the time-base correction for the rotational speed changes in the synchronous averaging procedure described by Huang and Wang (2005).

\section{Noise from the improved fan}

When the square inlet frame is replaced by the full-circle bellmouth and the large strut is trimmed down to form a set of four equal struts, as shown in Fig. 1(c), the only leading 
(a) Original fan

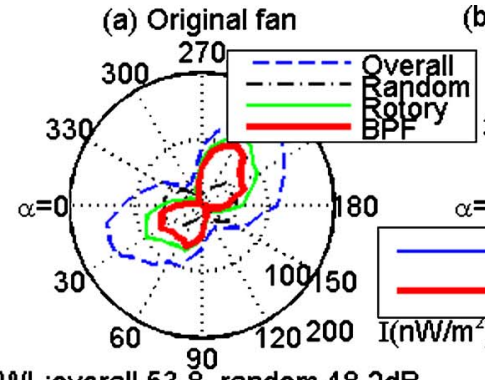

SWL:overall 53.8, random $48.2 \mathrm{~dB}$

(b) Original fan noise (BPF)

(d) Improved fan

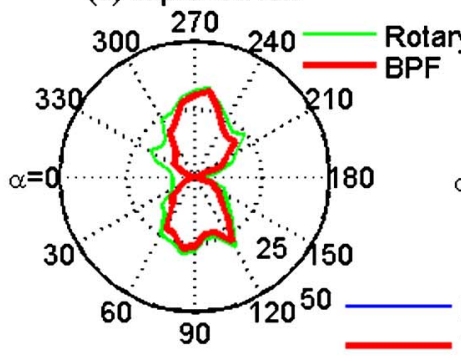

(e) Improved fan noise

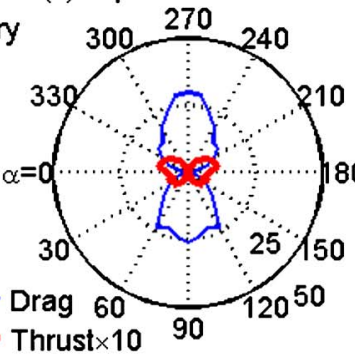

(c) Original fan, $\alpha=230^{\circ}$

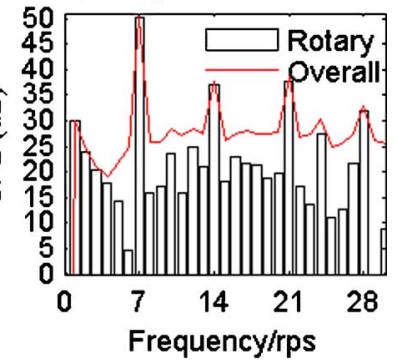

FIG. 2. (Color online) Comparison of sound intensity directivity and spectra from the original and the improved fans. (a) is the directivity of the original fan, (b) is the separation into the drag and thrust noise components, and (c) is the typical spectra at $\alpha=230^{\circ}$. (d), (e), and (f) are, respectively, the directivity, noise component separation, and spectra for the improved fan.

mode noise radiation comes from the drag component. The directivity of the measured sound intensity for the BPF is shown in Figs. 2(d) and 2(e). Since the random noise does not change much by the modifications, only the rotary noise is shown. Compared with the original fan, the total rotary noise is reduced from 52.4 to 48.5 , or by $3.9 \mathrm{~dB}$. The thrust noise power at the BPF, $S W L_{\mathrm{BPF}}^{\text {(thrust) }}$, is reduced from $45.6 \mathrm{~dB}$ for the original fan to $31.4 \mathrm{~dB}$ for the improved fan, which is a more significant reduction than that of the overall rotary noise. The rotary sound intensity shown in Fig. 2(d) is no longer tilted away from the rotational plane, and there is improved symmetry between the sound measured at $270^{\circ}$ and that at $90^{\circ}$. Using the method of noise source and modal decomposition of Eq. (3), the leading mode drag noise has $S W L_{\mathrm{BPF}}^{(D 1)}=48.1 \mathrm{~dB}$, while the higher order drag noise has $S W L_{\mathrm{BPF}}^{(D 2)}=31.2 \mathrm{~dB}$. The improved fan has a total BPF drag noise of $S W L_{\mathrm{BPF}}^{(\mathrm{drag})}=47.7 \mathrm{~dB}$, while that of the thrust noise is $16.3 \mathrm{~dB}$ below this level. As a result, the thrust noise can only be shown in Fig. 2(e) after being amplified by ten times. A typical SPL spectrum for $\alpha=90^{\circ}$ is shown in Fig. 2(f) for the improved fan. The first BPF peak is still about $20 \mathrm{~dB}$ above the broadband floor but, compared with the original fan, the improved fan is already very quiet and is chosen to be the starting point for the proposed active noise control scheme.

\section{NUMERICAL SIMULATION OF ANC}

The fan is represented by four unsteady drag forces distributed uniformly around the circumference with a rotating phase relation. The action of the active control is simulated by choosing a proper linear superposition of the two sound fields such that the pressure oscillation at the position of the error microphone is forced to be zero. All simulations are conducted for the fundamental blade passing frequency.

\section{A. Secondary source model}

The timing of the interaction is determined by the relative position between a rotor blade and the stationary struts. In this sense, the unsteady lift force occurs mainly when a rotor blade passes by a strut. As a result, the location is fixed relative to the stationary struts. However, as one blade comes to interact with a set of struts in a fixed sequence, the unsteady lift repeats from the position of one strut to the next with a fixed time delay, forming a pattern which can also be considered to be an oscillating force rotating continuously in space. Whether the source is better described by such a rotating force or fixed force with a rotating phase relation is purely a mathematical choice. Physically, the latter description is easier to model. The drag component of each interaction site, usually near the tip of a blade span, can be further decomposed into two parts, one in the horizontal direction, $F_{y}$, and another in the vertical direction, $F_{z}$, cf. Fig. 1(a). A normal fan has a set of evenly spaced rotor blades and struts; the summation of $F_{y}$ from all interaction sites can be simulated by a concentrated $F_{y}$ applied at the fan center. Since one oscillating force radiates an acoustic dipole, the difference between the distributed interaction forces and the total force located at the fan center represents two tightly coupled dipoles, or a quadrupole, which has a much smaller sound power and can be ignored in the current study. The same applies to $F_{z}$, and the result of all drag forces in all interaction sites can be represented by a pair of two forces at the fan center. The two component forces are

$$
F_{y}=A e^{i(\omega t+\pi / 2)}, \quad F_{z}=A e^{i \omega t}
$$

in which the $y$ component leads the $z$ component by $90^{\circ}$ as the fan rotates from the $+y$ axis towards the $+z$ axis, and $A$ is the amplitude. The sound radiated by each point force, 
(a) Linear amplitude of drag noise

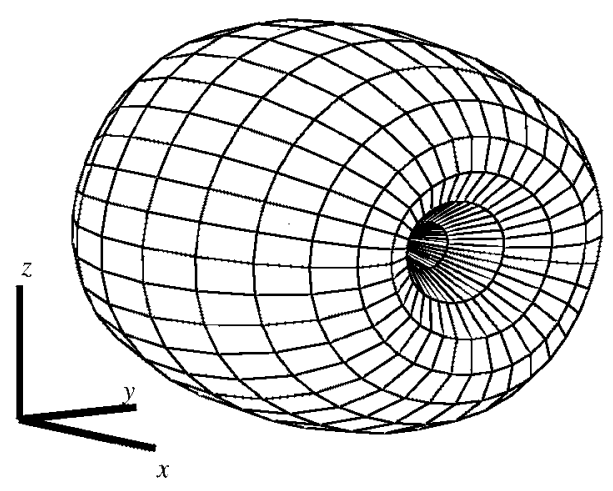

(c) Effect of error sensor position

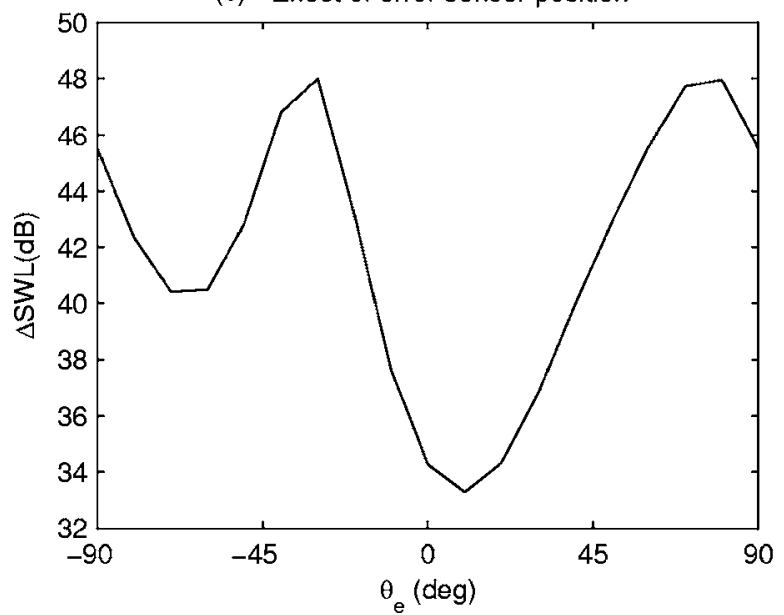

(b) Far field approximation

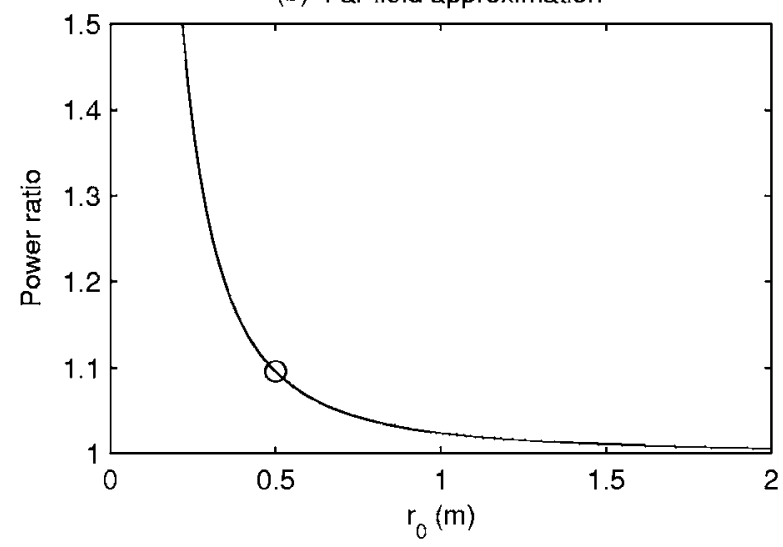

(d) Effect of loudspeaker mismatch

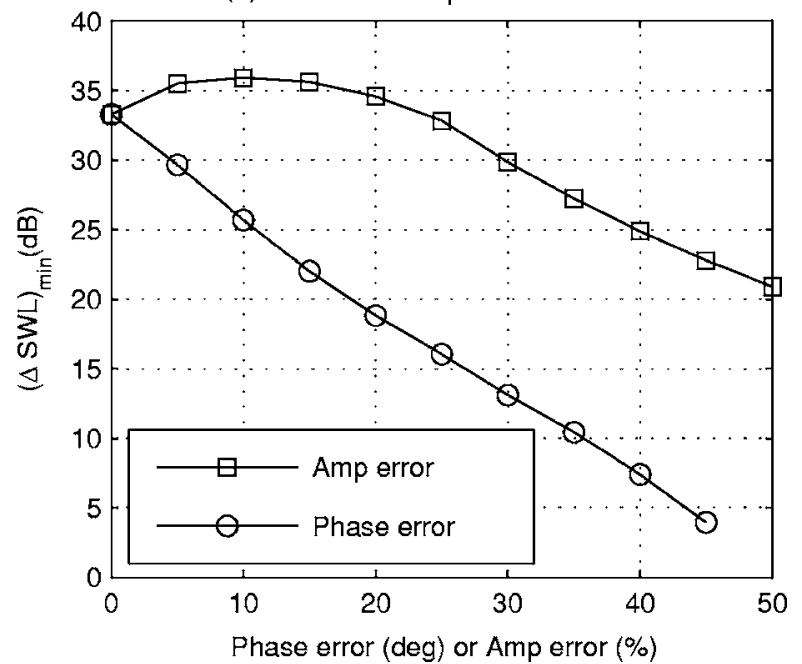

FIG. 3. Dipole simulations. (a) Fan noise simulated by four-point dipoles. (b) Ratio of the approximate to the accurate sound powers as a function of the measurement sphere radius. (c) The effect of the error microphone location on the control performance. (d) Effect of the loudspeaker phase and amplitude mismatch.

say $F_{z} e^{i \omega t}$, can be simulated by the following formulas (Dowling, 1998),

$$
\begin{aligned}
& \left.p\right|_{F_{z}}=\frac{i \omega \cos \theta}{4 \pi r_{0} c_{0}}\left(1+\frac{c_{0}}{i \omega r_{0}}\right) F_{z} e^{i \omega\left(t-r_{0} / c_{0}\right)}, \quad \cos \theta=\frac{z}{r_{0}} \\
& u_{r}=\frac{i \omega \cos \theta}{4 \pi \rho_{0} c_{0}^{2} r_{0}}\left(1+\frac{2 c_{0}}{i \omega r_{0}}-\frac{2 c_{0}^{2}}{\omega^{2} r_{0}^{2}}\right) F_{z} e^{i \omega\left(t-r_{0} / c_{0}\right)} \\
& u_{\theta}=\frac{\sin \theta}{4 \pi \rho_{0} c_{0} r_{0}^{2}}\left(1+\frac{c_{0}}{i \omega r_{0}}\right) F_{z} e^{i \omega\left(t-r_{0} / c_{0}\right)}, \quad u_{\alpha}=0
\end{aligned}
$$

where $p$ is the total oscillating pressure inclusive of propagating sound and the near field, $u_{r}, u_{\theta}, u_{\alpha}$ are the particle velocity in the radial, latitudinal, and longitudinal directions, respectively, and $\rho_{0}$ is the undisturbed density of fluid. The particle velocity is useful for the calculation of sound intensity and is not elaborated further. The sound generated by $F_{y}$ is obtained similarly. The only difference is that $z / r_{0}$, which derives from $\cos \theta$ in the first expression of Eq. (5), should be changed to $y / r_{0}$ for $\left.p\right|_{F_{y}}$. Together, the radiations by $F_{z}$ and $F_{z}$ form a rotating dipole whose pressure is given below,

$$
\begin{aligned}
& p=\left.p\right|_{F_{z}}+\left.p\right|_{F_{y}}=\frac{i \omega(z+i y)}{4 \pi r_{0}^{2} c_{0}}\left(1+\frac{c_{0}}{i \omega r_{0}}\right) A e^{i \omega\left(t-r_{0} / c_{0}\right)}, \\
& |z+i y|=r_{0} \sin \phi \\
& |p|=\frac{A \omega \sqrt{1+\left(\omega r_{0} / c_{0}\right)^{-2}}}{4 \pi r_{0} c_{0}} \sin \phi .
\end{aligned}
$$

The rotating dipole has the directivity of $|p| \propto \sin \phi$, which has the shape of a ring around the $x$ axis, which is given in Fig. 3(a).

The above simulation results are obtained by placing the two forces at the same point which is meant to be the fan center. Experimentally, each component dipole is realized by a loudspeaker with a finite size. In fact, it is impossible to place the two loudspeakers at the fan center position without seriously blocking the flow. Under such structural constraints, one pair of loudspeakers is put on the top edge of the fan frame and another pair under the bottom edge of the fan frame. This arrangement is illustrated in Fig. 4(b) and the photo is shown in Fig. 4(c). The two pairs approximate a rotating dipole placed at the center of the fan. Numerical simulation is easily conducted to see the difference between 


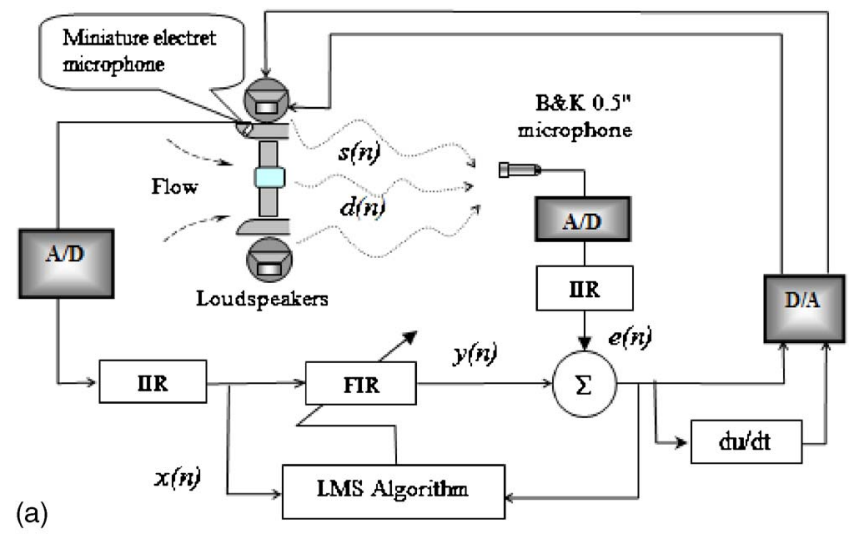

(a)
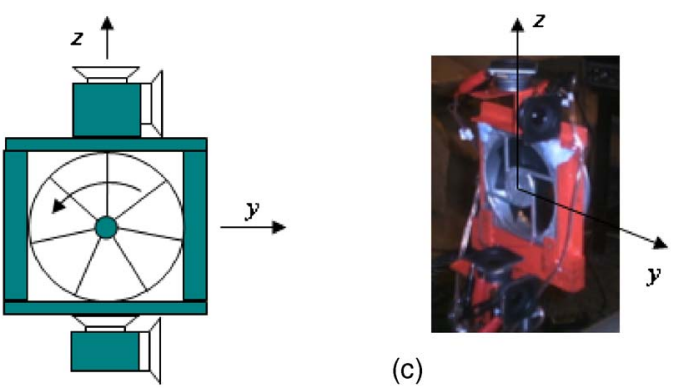

(c)

FIG. 4. (Color online) Experimental setup. (a) Overall view of the control system. (b) Schematic of the secondary source arrangement. (c) The back view (photo) of the two pairs of loudspeakers, two horizontal and two vertical.

the sounds from the two pairs of loudspeakers and a single rotating dipole with perfect source collocation with the fan center. The difference is found to be negligible for the parameters relevant to the current experiment.

The dipole sound has both far-field and near-field components, and the exact sound intensity should be calculated as $I=\frac{1}{2} \operatorname{Re}\left(p u_{r}^{*}\right)$, where $u_{r}^{*}$ is the conjugate of the radial component of the acoustic particle velocity. In the experiment, however, a simple measurement of the free field takes only the local pressure. The far-field approximation, $I=p_{\mathrm{rms}}^{2} /\left(\rho_{0} c_{0}\right)$, is used for sound intensity calculation, where $p_{\text {rms }}$ is the local root-mean-square value of the measured pressure oscillation. Ideally, the measurement microphone is placed as far away as possible from the source, but a close proximity is necessary if the source is weak and a good signal-to-(electronic) noise ratio is desired. The percentage error caused by the far-field approximation for the BPF of $350 \mathrm{~Hz}$ is simulated by the dipole shown in Fig. 3(a), and the result is shown in Fig. 3(b). The compromise reached in the current experiment is to place the error microphone at $r_{0}$ $=0.5 \mathrm{~m}$ from the fan center, and the expected deviation in sound power estimation is $9.6 \%$ or $10 \log _{10}(1.096)=0.4 \mathrm{~dB}$, which is marked by an open circle in Fig. 3(b).

\section{B. Optimization of the error microphone position}

The secondary source is driven by a signal produced in such a way that the sound radiated cancels exactly the primary noise at the position of the error microphone. The sound radiated by a single loudspeaker follows Eq. (5), while that by a rotating dipole follows Eq. (6). The signal obtained at the error microphone is used to adjust the signal fed to the secondary source such that the combination of the fan noise, say $p_{\text {fan }}$, and the antisound, $C p_{\text {anti }}$, cancel at this point, $p_{\text {fan }}$ $+C p_{\text {anti }}=0$, where $p_{\text {anti }}$ is the antisound generated by the two pairs of tightly coupled loudspeakers at the position of the error microphone by a unit voltage amplitude, and $C$ is the complex amplitude to be determined either manually in an open loop control, or automatically in a closed-loop control. The position of the error microphone affects the global control results, and many strategies exist to place the error microphone or microphones so that a certain global measure of residual noise is minimized. In the current problem, the primary noise to be controlled has been shown to have the directivity of $p_{D 1} \propto \sin \phi$, so a single error microphone position can be used. The primary noise is simulated by four circumferential point forces at an equal angular interval of $90^{\circ}$ and the source radius is $r_{s}=5 \mathrm{~cm}$, which is about $83 \%$ of the fan radius of $6 \mathrm{~cm}$. The first source is placed on the $+y$ axis at $x=z=0, y=r_{s}$. The four antisound loudspeakers are simulated by two rotating dipoles, one above the fan at $z$ $=10 \mathrm{~cm}$, and one below the fan at $z=-10 \mathrm{~cm}$, both with $x$ $=y=0$. For obvious reasons, the error microphone is chosen to be located on the rotational plane of $x=0$ or $\phi=90^{\circ}$. The exact location is further specified by the angular value denoted by $\theta_{e}$, for which $\theta_{e}=0,+90^{\circ},-90^{\circ}$ represents the intersection points of the observer sphere with the $+z,+y,-y$ axes, respectively. The total sound power reduction, $\Delta S W L$, is found as a function of $\theta_{e}$, as shown in Fig. 3(c). The variation curve is asymmetrical but the asymmetry vanishes when the radial position of the error microphone, $r_{0}$, is increased towards infinity. In other words, the asymmetry is caused by the near-field effect. For $r_{0}=0.5 \mathrm{~m}$, the optimal angular position for the error microphone is found to be close to the horizontal plane position of $\theta_{e} \approx 90^{\circ}$, but in fact the angular position is relative to the first source force position, which is not actually known in experiment. However, the lowest value of sound power reduction, $\triangle S W L$ $=33.3 \mathrm{~dB}$, is expected to be realized in experiment.

\section{Effect of loudspeaker mismatch}

Two loudspeakers are used to construct one rotating dipole as antisound. In order to simplify the control rig, only one output signal is used. The signal is used to drive one loudspeaker, say the one facing the horizontal direction. The time derivative of this signal is used to drive the other loudspeaker in order to make sure that the two form a $90^{\circ}$ phase difference. Such configuration is based on the assumption that the two loudspeakers behave identically. The fact that there is bound to be some difference in their response to input signals calls for amplitude and phase correction. A fixed amount of correction can be embedded in the control circuit without difficulty. However, such correction cannot account for variations in loudspeaker performance. Simulation is thus conducted to see the sensitivity of the loudspeaker mismatch and the global noise suppression performance. The minimal sound power reduction obtained for the worst error microphone position relative to the source location is used. In other words, the trough of the curve in Fig. 3 (c) is used for a given configuration. The configuration is 
then changed to one in which the two loudspeakers facing the vertical directions are given an amplitude or phase angle mismatch with the response of the two loudspeakers facing the horizontal direction. The variations in amplitude and phase are tested separately, and the resulting minimal sound power reductions are plotted together in Fig. 3(d). Both curves begin with the value of $33.3 \mathrm{~dB}$ for the reference configuration in which the two loudspeakers are identical in responses. For the performance of $\Delta S W L$ to deteriorate to $20 \mathrm{~dB}$, the amplitude mismatch should be roughly $50 \%$ (end of the upper curve), while the phase mismatch is close to $20^{\circ}$. It may be said that the performance is more sensitive to phase mismatch. During the actual experiment lasting for one hour or so, the loudspeaker is found to vary in amplitude within a band of around $30 \%$, while the phase variation is around $15^{\circ}$. The results of this simulation seem to indicate that both are tolerable as far as a target of around 20-dB sound power reduction is concerned.

In order to obtain the best result using the close-loop control, multi-channels may be necessary. In this case, the loudspeakers facing the horizontal direction control the horizontal component of the rotating dipole, and their input signals should be adjusted by signals from an error microphone located on the central horizontal plane. The second channel for the two vertical-facing loudspeakers should be adjusted by an error microphone placed at the top of the fan. Both error microphones should be placed on the rotational plane. The use of multi-channel control is beyond the scope of the current study which is entirely motivated by the pursuit of simplicity and practicality of a possible active control method.

\section{ACTIVE CONTROL STUDIES}

As shown by Huang and Wang (2005), the configuration of $B=7$ blades with $S=4$ struts features rotating dipoles for the fundamental BPF tone and the third harmonic, while the second harmonic is in the higher spinning pressure mode with $\nu=2$. The latter can be seen as a set of tightly coupled dipoles or an approximate quadrupole. The sound power levels for the three harmonics are experimentally found to be 47.8, 32.7, and $35.4 \mathrm{~dB}$, respectively. The second harmonic is indeed low and is not much of a concern here, while the third harmonic is higher than the second due to the leading mode radiation. However, its absolute level is also not very high and is left out of the control scheme.

\section{A. Adaptive and open-loop feed-forward control}

The experimental setup for the closed-loop control is shown in Fig. 4(a) schematically. The reference signal is provided by a miniature electret microphone (151 series supplied by Tibbet industry) located on the bellmouth of the fan. It senses the near-field aerodynamic pressure on the bellmouth surface, and the signal is a saw-tooth-like waveform, which has a richer BPF content than the narrow pulses provided by a photoelectric tachometer, the difference being around $20 \mathrm{~dB}$. The electret microphone has a flat frequency response of $0.018 \mathrm{~V} / \mathrm{Pa}$ from $300 \mathrm{~Hz}$ to $5 \mathrm{kHz}$. This sensitivity is very low compared with that of the condenser-type

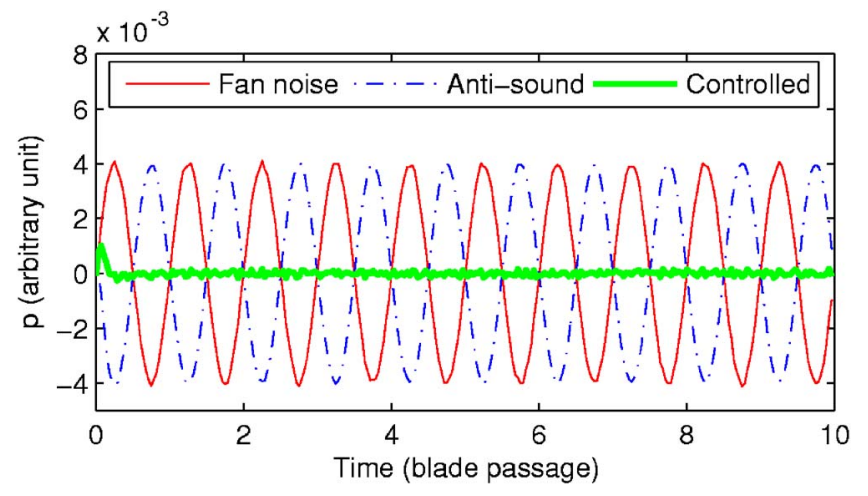

FIG. 5. (Color online) The computer simulation of the LMS control algorithm.

microphone used for the directivity measurement. In other words, the aerodynamic pressure oscillation sensed is much higher than the radiated sound, eliminating a possible feedback path in the control rig. The reference signal is bandpass filtered to keep the components of the BPF tone. A six-order Chebyshev infinite-impulse-response (IIR) filter is constructed by using the least $p$-norm optimal IIR filter design in the SIMULINK of MATLAB ${ }^{\circledR}$ (Wang et al., 2005). The error signal is taken by a half-inch $\mathrm{B} \& \mathrm{~K}$ microphone located at $x=z=0, y=0.5 \mathrm{~m}$, which is level with the fan center on the rotational plane. A time-domain adaptive filtered- $X$ LMS feedforward controller (described below) constructs the antisound signal to drive the secondary sound sources, which are made by the 2-in. loudspeakers. The output signal from the LMS circuit is divided into two paths, one direct path, which drives the horizontally oriented loudspeaker, and another with a time derivative, which gives a $\pi / 2$ phase delay to drive the vertically oriented loudspeaker. Together, they form a dipole rotating counter-clockwise, simulating the drag noise from the fan rotating in the same direction. Physically, these two loudspeakers cannot be located at the center of the cooling fan. They can only be located outside the casing of the fan, and the result would be two not-so-tightly-coupled dipoles with their centers away from the fan center. In order to align the antisound with the fan noise source, two pairs are used, one above and one below the center of the fan. The back view of the fan equipped with four loudspeakers is shown in Fig. 4(c) and the schematic for this arrangement is shown in Fig. 4(b).

A 16-order normalized LMS adaptive filter is adopted. The FXLMS algorithm is tested in the SIMULINK and the result is shown in Fig. 5. The disturbance is a harmonic signal of $357 \mathrm{~Hz}$ plus a random noise of $3 \%$ in amplitude, which is around $30 \mathrm{~dB}$ below the main disturbance signal. The convergence is achieved after only $\frac{1}{4}$ of a period, or seven floating data points. The cancellation is about $30 \mathrm{~dB}$, which means that the main disturbance signal is eliminated. The purpose of this simulation is merely to check the precision and the convergence speed of the control algorithm. The actual convergence speed and the mean square error depend on many physical parameters such as the physical control path transfer function. The control algorithm is built on a dSPACE (DS1103 PPC) controller, which is a real-time system with multiple $\mathrm{A} / \mathrm{D}$ and $\mathrm{D} / \mathrm{A}$ channels, and a Motorola 
PowerPC 604e microprocessor running at $333 \mathrm{MHz}$, which is connected to a personal computer through an ISA bus. A real time interface (RTI) is used to build the code downloaded to and executed on the dSPACE hardware. The rotational reference signal and the error microphone signal are sampled at $10 \mathrm{kHz}$, and the output analog signal is also constructed at an update rate of $10 \mathrm{kHz}$; both are the upper limit of the DS1103 PPC controller board.

The technique of the open-loop control was applied to the thrust noise control (Wang et al., 2005) and it succeeded in giving a total of 10.8- $\mathrm{dB}$ rotary sound power reduction. Considering the prospect of practical implementation for a cooling fan, the open-loop control is also tested here to see how its performance compares with that of the closed-loop described above. In the open-loop control, the same reference signal from the electret microphone is used. The signal is simply band-pass filtered, phase delayed, and amplified to drive the secondary loudspeaker. The phase delay and amplification are manually adjusted by using an error microphone located on the rotational plane. Once the two parameters are tuned, they are fixed in the control algorithm. For the current application of drag noise control, two output channels are used to drive two loudspeakers in one pair. The primary output drives the horizontal loudspeaker while the channel with the time derivative as described earlier drives the vertical loudspeaker. Of course, the second output channel is fixed to the first, but it can have its own fixed amount of time delay and independent amplification to account for the differences in the loudspeaker responses. The finding of the second channel parameters has to rely on the use of a second error microphone located on the $+z$ axis where the first loudspeaker does not radiate sound. In fact, these compensatory parameters are also used in the closed-loop control. Since, in reality, two pairs of loudspeakers are used, one channel drives two horizontal loudspeakers and another does the two vertical loudspeakers.

The acoustic directivity and the integrated sound powers before and after the control are measured at a spherical radius of $r_{0}=0.5 \mathrm{~m}$ by a second measurement microphone (B\&K type 4187) not shown in Fig. 4(a). The signal from the measurement part of the instrumentation has no involvement in the control circuit. The acoustic directivity is measured on the central horizontal plane level with the fan center, $z=0$ or $\theta=\pi / 2$. The synchronously averaged sound is used to calculate the far-field approximation of the sound intensity, $I$ $\approx p_{\text {rms }}^{2} / \rho_{0} c_{0}$, where $p_{\text {rms }}$ is the rms value of the BPF component of sound. A total of 36 points are measured with an angular interval of $10^{\circ}$, and the sound power is calculated by (Huang and Wang, 2005)

$$
\begin{aligned}
W & =2 \pi r_{o}^{2} \int_{0}^{\pi / 2} I(\alpha)|\sin (\alpha)| d \alpha \\
& \approx \pi^{2} r_{o}^{2}(\Delta \alpha) \sum_{i=1}^{36} I\left(\alpha_{i}\right)\left|\sin \left(\alpha_{i}\right)\right| .
\end{aligned}
$$

To evaluate the global effectiveness of the control, directivity measurement is also conducted for another two horizontal (a) Close-loop control
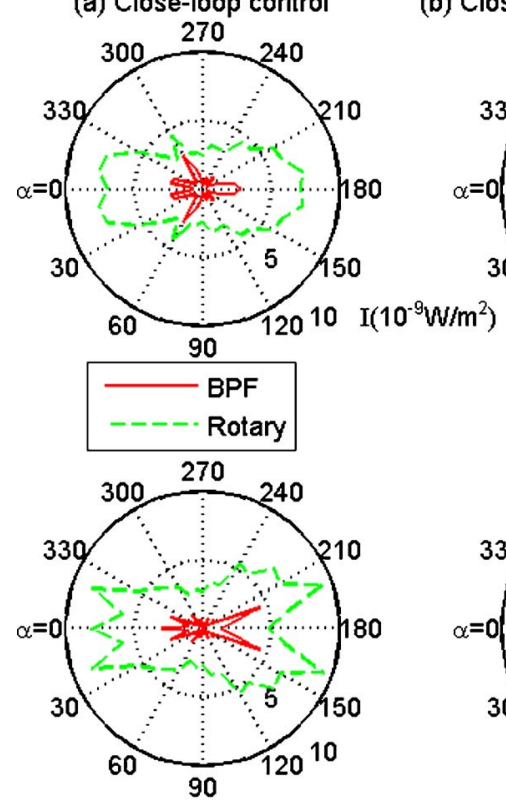

(c) Open-loop control (b) Close-loop source decomposition

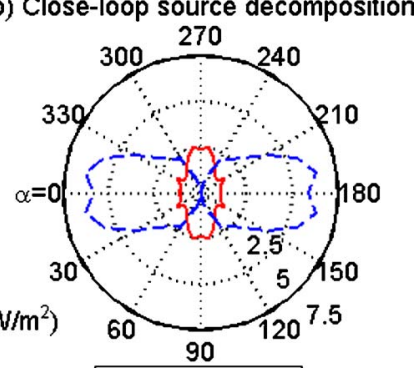

FIG. 6. Control-on sound intensity directivity for the close-loop (a,b) and open-loop $(\mathrm{c}, \mathrm{d})$ schemes with source decomposition analyses $(\mathrm{b}, \mathrm{d})$.

planes of $\theta=\pi / 3, \pi / 6$ together with the top point of $\theta=0$, cf. Fig. 1(a).

The results are presented in two parts in the next two subsections. In the first, directivity patterns for the control-on and control-off are compared for the central horizontal plane $(\theta=0, z=0)$. The results for the closed-loop and the openloop controls are compared. The comparison shows that the two control algorithms give very similar results. So, in the second part, the results of the three-dimensional measurement for the whole observer sphere is conducted for the open-loop control.

\section{B. Directivity results and analyses}

Figure 6 shows the results of the directivity measurement on the central horizontal plane, which should be studied together with the control-off measurements shown in Fig. 2. Figures 6(a) and 6(b) are for the closed-loop control and Figs. 6(c) and 6(d) are for the open-loop control. Figure 6(a) shows that the total rotary noise (outer dashed curve) is mainly aligned in the axial direction, which implies thrust noise and contrasts with the dominant drag noise pattern of Fig. 2(d). The BPF component, shown as the inner solid line in Fig. 6(a), is subject to control and it has a rather irregular shape, meaning that the residual noise is small. Assuming that there is a symmetry on the rotational plane, the sound power reductions, denoted as $\Delta S W L$, from the control-off to the control-on states are found as follows,

$$
\begin{aligned}
& \Delta S W L_{\mathrm{BPF}}^{(\mathrm{drag})}=47.4-32.0=15.4 \mathrm{~dB}, \\
& \Delta S W L_{\mathrm{All}}^{(\mathrm{drag})}=48.0-37.4=10.6 \mathrm{~dB}, \\
& \Delta S W L_{\mathrm{BPF}}^{\text {(thrust) }}=31.4-32.5=-1.1 \mathrm{~dB},
\end{aligned}
$$




$$
\Delta S W L_{\text {All }}^{\text {(thrust) }}=39.1-38.2=0.9 \mathrm{~dB},
$$

where subscripts "All" imply all harmonics of the BPF. Note that the thrust noise is not subject to any active control and its level varies slightly between the control-on and controloff conditions. The results of the open-loop control shown in Figs. 6(c) and 6(d) are very similar to Figs. 6(a) and 6(b). Again, the residual rotary noise pattern is irregular, implying that the drag noise abatement by the active control is quite complete.

It can be concluded that the drag noise reduction for the BPF achieved in this study, which is around $15-16 \mathrm{~dB}$, validates the basic principle pursued in this study, but it is well below the theoretical prediction of about $33 \mathrm{~dB}$. The performance is apparently limited by factors not considered in the theoretical prediction. In the previous study of thrust noise control using a smaller cooling fan (Wang et al., 2005), it was found that the effect of the variation of the rotational speed from one cycle to the next is negligible. This conclusion is reexamined for this study and is also found to be valid. The factor of mismatch between two loudspeakers used in a pair for rotating dipole is also excluded by the following considerations. Numerical simulation for the acoustic interference, Fig. 3(d), shows that at least $20 \mathrm{~dB}$ sound power reduction is achieved even when the two loudspeakers mismatch in their phase by about $20^{\circ}$ or in their amplitudes by about $50 \%$. Observations during experiment show that the two pairs of loudspeakers chosen have much less mismatches.

Two more clues for the ultimate performance limitation are analyzed below. The first is the variation of sound radiation by the cooling fan when its rotational speed is held absolutely constant. This variation may have its origin in turbulent flow aerodynamics, and it has also been analyzed previously (Wang et al., 2005). The algorithm of all active fan noise control takes the signal from one blade passage to construct the antisound for the sound radiated in the time period of the next blade passage. The delay can be longer if the error microphone is placed in a distant far field. The apparently turbulent variation in sound radiation from the fan represents the part of uncontrollable noise. To analyze this effect, the time-domain signals from the whole central horizontal plane are analyzed in terms of the BPF amplitude variation from one blade passage to the next, and the result shows that, on average, $\sigma=10.0 \%$ of amplitude variation is found all around the fan. The difference between the controllable and uncontrollable sound power is thus estimated as

$$
\Delta S P L=-20 \log _{10}(\sigma) \underset{\sigma=0.10}{\longrightarrow} 20.0 \mathrm{~dB} .
$$

This value is very close to the number of sound pressure reductions achieved at the error microphone position, where, theoretically, the sound is supposed to be completely cancelled if all sound radiation is deterministic.

The second possible performance limitation is analyzed as follows. The sound pressure level reduction of around $20 \mathrm{~dB}$ at the error microphone position indicates that the basic control strategy is sound. The fact that both closed- and open-loop controls manage to achieve similar performance in this regard means that the system is essentially time station- ary, and the additional capability of the adaptive control has not shown its potential benefit. The flaw in the control rig then must lie in the lack of perfect acoustic directivity match between the reality and the ideal distribution of $p_{D 1} \propto \sin \phi$ for the leading mode drag noise. Factors of nonideal sound radiation include higher order drag noise featuring $p_{D 2}$ $\propto \sin ^{2} \phi$, cf. Eq. (2). Assume now that the measured drag noise is a sum of the two in the form of

$$
p=p_{1} \sin \phi+p_{2} \sin ^{2} \phi,
$$

where the magnitudes of each mode can actually be found by the source and mode decomposition method designed by Huang and Wang (2005), but their phase relation is not known. When the control is applied at one point, the sound power $W$ becomes

$$
\begin{aligned}
W= & \int_{0}^{\pi}\left(2 \pi r_{0}^{2} \sin \phi\right) I d \phi \\
= & 2 \pi r_{0}^{2}\left(\rho_{0} c_{0}\right)^{-1} \int_{0}^{\pi} \sin \phi\left(p_{1} \sin \phi+p_{2} \sin ^{2} \phi\right)^{2} d \phi \\
= & \underbrace{2 \pi r_{0}^{2}\left(\rho_{0} c_{0}\right)^{-1}}_{\equiv C_{w}} \int_{0}^{\pi} \\
& \times\left(p_{1}^{2} \sin ^{3} \phi+2 p_{1} p_{2} \sin ^{4} \phi+p_{2}^{2} \sin ^{5} \phi\right) d \phi \\
= & C_{w}\left(\frac{2}{3} p_{1}^{2}+\frac{3 \pi}{8} p_{1} p_{2}+\frac{8}{15} p_{2}^{2}\right) .
\end{aligned}
$$

When the error microphone is located at $\phi=\pi / 2$ where the two modes add up, $p_{\phi=\pi / 2}=p_{1}+p_{2}$, the secondary sound field is given as $p_{s}=-p_{\phi=\pi / 2} \sin \phi$ and the residual noise $p_{\text {res }}$ and its sound power are found as follows,

$$
\begin{aligned}
& p_{\text {res }}=p_{s}+p=-p_{2} \sin \phi+p_{2} \sin ^{2} \phi, \\
& W_{\text {res }}=C_{W}\left(\frac{2}{3}-\frac{3 \pi}{8}+\frac{8}{15}\right) p_{2}^{2}=C_{W} 0.0219 p_{2}^{2} .
\end{aligned}
$$

When the error microphone is located where the first and second mode sounds cancel, $\phi=3 \pi / 2$, the residual sound and its power, both denoted by a subscript "res," are found to be

$$
\begin{aligned}
& p_{\text {res }}=p_{s}+p=+p_{2} \sin \phi+p_{2} \sin ^{2} \phi, \\
& W_{\text {res }}=C_{W}\left(\frac{2}{3}+\frac{3 \pi}{8}+\frac{8}{15}\right) p_{2}^{2}=C_{W} 2.3781 p_{2}^{2} .
\end{aligned}
$$

The above two scenarios represent two extreme cases. The second case is the worst result, and its sound power compares with the second mode drag noise, denoted by a subscript " 2 ," as follows,

$$
\begin{aligned}
& \frac{W_{\text {res }}}{W_{2}}=\frac{C_{W} 2.3781 p_{2}^{2}}{C_{W}(8 / 15) p_{2}^{2}}=4.4589, \\
& S W L_{\text {res }}-S W L_{2}=10 \log _{10}(4.4589)=6.5 \mathrm{~dB} .
\end{aligned}
$$




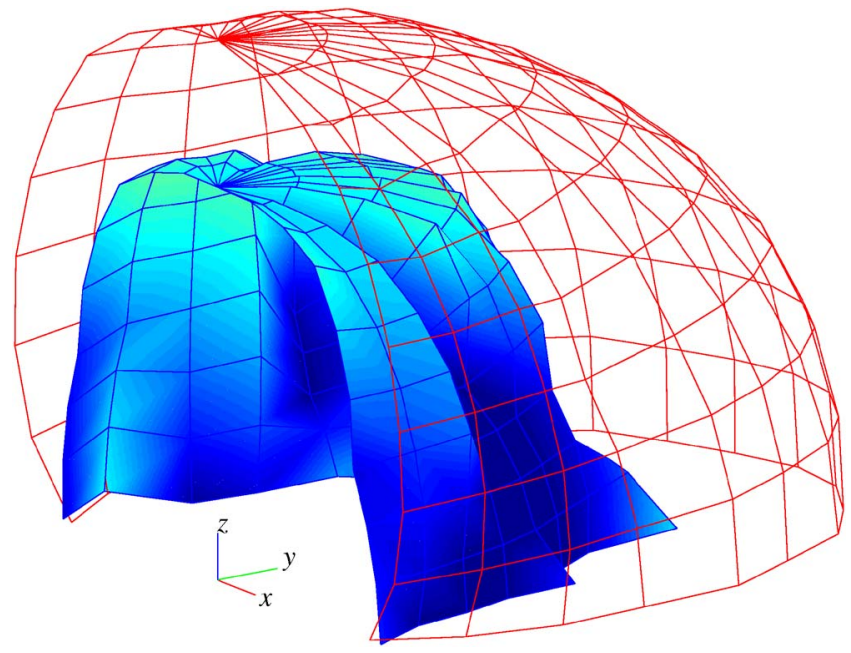

FIG. 7. (Color online) Comparison of the experimental data of the sound pressure level for the control off (outer wire-mesh) and control on (inner surface) configurations for one-quarter of the observation sphere.

The difference of $6.5 \mathrm{~dB}$ represents the intermodal coupling which cannot be tackled by the single rotating dipole control method, which only deals with the leading mode drag noise.

When the measured directivity of Fig. 2(d) is analyzed for the second-order drag noise, it is found that its sound power level is $S W L_{\mathrm{BPF}}^{(D 2)}=31.2 \mathrm{~dB}$. The final residual drag sound power is $37.4 \mathrm{~dB}$ for the closed loop and $37.9 \mathrm{~dB}$ for the open loop. These represent 6.2 and $6.7 \mathrm{~dB}$ above $S W L_{\mathrm{BPF}}^{(D 2)}$, which are, incidentally, very close to the upper limit of $6.5-\mathrm{dB}$ intermodal coupling error. It must be emphasized, however, that the above analysis has many implicit assumptions about other aspects of the control rig, and the quantitative coincidence must be treated cautiously.

\section{Global noise reduction}

Three $\frac{1}{2}$-in. B\&K microphones provide simultaneous directivity measurement for the three horizontal cross sections of $\theta=30^{\circ}, 60^{\circ}, 90^{\circ}$ on the observer sphere of $r_{0}=0.5 \mathrm{~m}$, cf. Fig. 1(a). Due to the physical limitations, the error microphone is placed at a level slightly higher than the fan center, but it is still on the rotational plane. A total of 36 points are measured for each horizontal plane with an angular interval of $\Delta \alpha=10^{\circ}$. The data of the three planes, $\theta=30^{\circ}, 60^{\circ}, 90^{\circ}$, are used to calculate the total sound power radiation by the fan by assuming a perfect symmetry of the upper $(+z)$ and lower $(-z)$ hemisphere,

$$
\begin{aligned}
W & =2 \int_{0}^{\pi / 2} r_{0}^{2} \sin \theta\left[\int_{0}^{2 \pi} I(\alpha, \theta) d \alpha\right] d \theta \\
& \approx 2(\Delta \alpha \Delta \theta) r_{0}^{2} \sum_{n=1}^{3} T_{n} \sin \theta_{n} \sum_{m=1}^{36} I\left(\alpha_{m}, \theta_{n}\right),
\end{aligned}
$$

where $T_{n}=1,1,0.5$ for $\theta_{n}=30^{\circ}, 60^{\circ}, 90^{\circ}$ are the weighting coefficients for the numerical integration following the trapezoidal rule. Note that the plane of $\theta=0$ is reduced to one point on the top of the sphere with $\sin \theta=0$; it makes no contribution to the numerical summation and is left out. In order to plot the results in three-dimensional view
TABLE I. Changes in sound power levels (all in $\mathrm{dB}$ re $10^{-12} \mathrm{~W}$ ).

\begin{tabular}{lccc}
\hline \hline Sound power level & Control off & Control on & Reduction \\
\hline Random noise & 49.6 & 49.8 & -0.2 \\
Rotary total & 48.5 & 41.4 & 7.1 \\
Rotary BPF & 47.8 & 35.5 & 12.3 \\
Drag noise BPF & 47.8 & 34.8 & 13.0 \\
\hline \hline
\end{tabular}

smoothly, the sound intensity is further interpolated from three to nine horizontal mesh sections of $\theta$ $=10^{\circ}, 20^{\circ}, \ldots, 90^{\circ}$, where $\theta=0$ also provides one data point for interpolation. The comparison of the BPF drag noise for the conditions of control-on and control-off is made in Fig. 7, where only one quadrant of the 3D directivity for sound pressure level is given. This figure confirms that the noise suppression for the rotating dipole is global in nature. Note that the noise is not reduced along the rotational axis where thrust noise peaks and the applied control has, theoretically, no effect.

The numerical comparisons for the sound power levels for various components are given in Table I. As shown in the first row of Table I, the random noise hardly changes; in fact, it increases by $0.2 \mathrm{~dB}$. The focus is on the tonal noise, namely the rotary noise in the current context. Looking down the first column for the control-off state, it is found that the rotary noise is mainly dominated by the BPF component, which is in turn dominated by the drag noise. Note that there is a second decimal point difference between the rotary noise and the drag noise. For the control-on state, the second column shows less dominance by the drag noise in the total rotary noise since the drag noise is suppressed by the active control scheme. The direct objective of the control is the BPF component of the drag noise, which is reduced by $13.0 \mathrm{~dB}$, as shown in the last row of the table. The total rotary noise is decreased by $7.1 \mathrm{~dB}$, which is much less impressive than the drag noise reduction since it contains all frequencies and all noise mechanisms. In terms of the rotary noise for the first BPF, it is reduced by $12.3 \mathrm{~dB}$, which is very close to the $13.0 \mathrm{~dB}$ reduction for the drag noise. Notice that the sound power reduction for the rotary BPF is about $3 \mathrm{~dB}$ more than that calculated by the data for the central horizontal plane alone. This could be attributed to the fact that the position of the error microphone has changed a little during the measurements.

Figure 8 gives the spectral comparison between the configurations of control-on and control-off. The three subfigures are for the three rotational plane points $\left(\phi=90^{\circ}\right)$ on the three measurement planes of $\theta=30^{\circ}, 60^{\circ}, 90^{\circ}$. The sound pressure level reductions for the BPF are 10.7, 18.6, and 20.6 dB for the three positions, respectively.

\section{CONCLUSIONS}

The findings of this work are summarized before comparison is made with the thrust noise control reported in Wang et al. (2005).

(1) A typical computer cooling fan available in the market is very noisy due to two gross features of the 

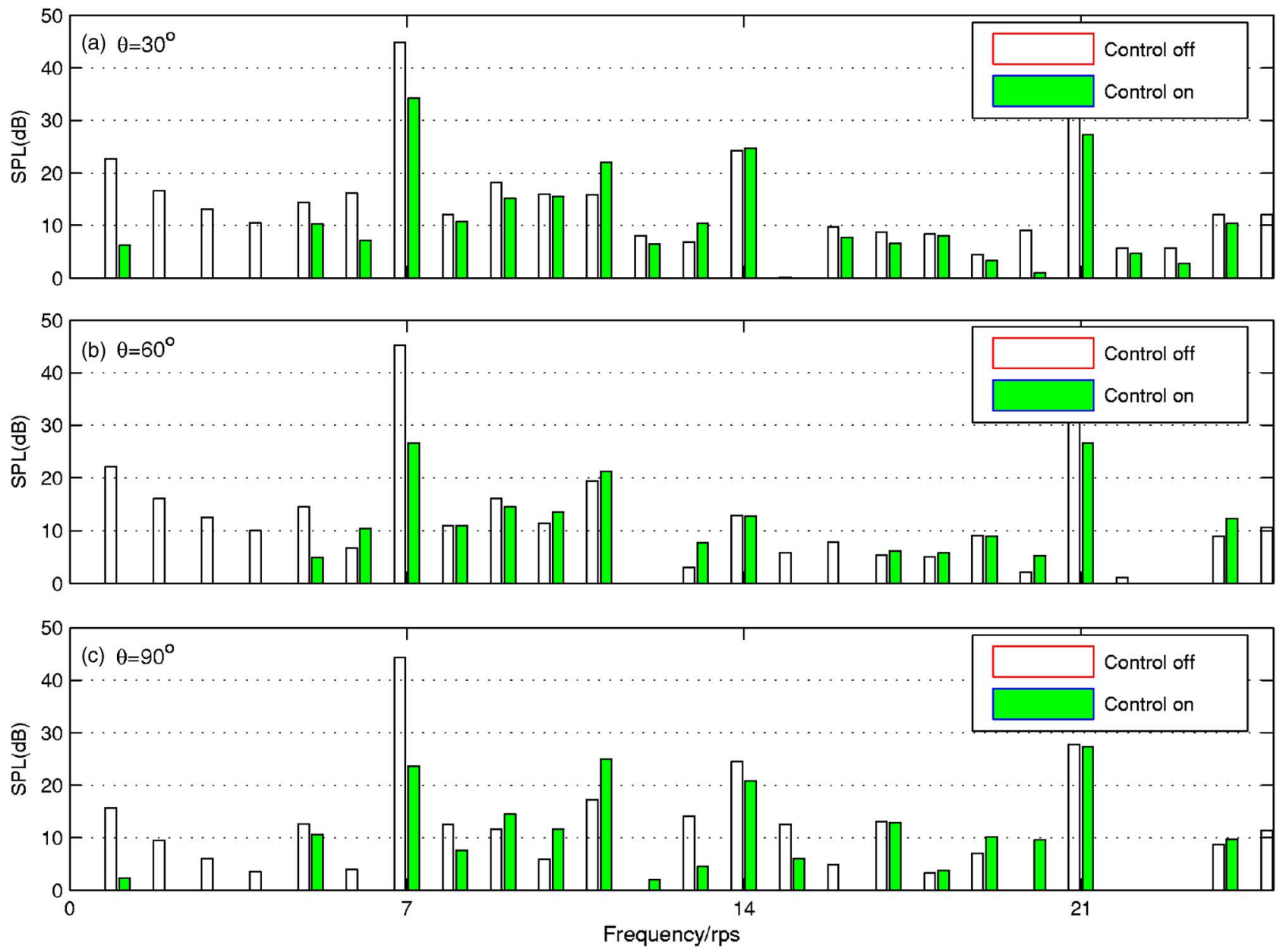

FIG. 8. (Color online) Spectral comparison for the control off (open bars) and control on (filled bars) for three points on the rotational plane $(\phi=\pi / 2)$.

structural design. One is the square frame which distorts the inlet flow to form a four-lobe pattern. The other is the wire-carrying strut which is a powerful noise source for both drag and thrust components. When these two features are corrected, the rotary sound power is reduced by about $4.2 \mathrm{~dB}$. The measurement of the noise radiated by the improved fan shows a very ideal rotating dipole pattern, with an outstanding BPF peak at about $20 \mathrm{~dB}$ above the broadband of the spectrum.

(2) Based on the point force approximation, drag noise is radiated by the dipole source rotating with rotor blade around the rotational plane. The numerical simulation decomposes the rotating point force into two components with a rotating phase relation. Experimentally, a pair of two loudspeakers can be used to construct the rotating dipole when the two are installed perpendicular to each other with a $90^{\circ}$ phase difference. In the current study, two such pairs are used in order to collocate the secondary sources with the fan center. The antisound approximates the primary fan noise very well, and a power reduction of $13.0 \mathrm{~dB}$ for the drag noise is achieved at the blade passing frequency. However, higher order drag noise also exists, and this component is beyond the scope of the current active control scheme.
(3) Fan noise radiation varies from one blade passage to the next in a random fashion even when the rotational speed is held constant, and the linear amplitude variation is found to be about $10 \%$. Since the antisound is constructed from the signal input from the previous blade passage of the rotation, such random variation would cause incomplete noise cancellation. The measured maximum pointwise noise reduction of around $20 \mathrm{~dB}$ at the position of the error microphone is compatible with this hypothesis of the performance limitation. The mechanism might be rooted in the turbulent nature of the aerodynamic process.

(4) Higher order drag noise exists due to many factors, such as the difference between the fluctuation forces arising from the rotor-strut encounters between different rotor blades and struts due to either aerodynamic uncertainties or structural imperfections. The first higher order drag noise features a spinning pressure mode of $|\nu|=2$, and its sound pressure directivity is $\sin ^{2} \phi$, which means that the sound pressure on the opposing sides on the rotational plane are the same, $\sin ^{2} \phi=\sin ^{2}(\phi+\pi)$. This differs from the leading mode drag noise with a pressure directivity of $\sin \phi$, which is antisymmetric across the rotational plane. The existence of a higher order drag noise component 
would lead to the excess leading mode antisound determined by the pressure minimization procedure at the error microphone position. The coupling of this excess leading mode antisound and the higher order drag noise could lead to a maximum of $6.5-\mathrm{dB}$ amplification of the higher order drag noise. The analysis of the control results indicates that the actual performance is close to this maximum overshoot, and this could be the ultimate limitation factor for the active drag noise control using the leading mode rotating dipole.

(5) Both open- and closed-loop (adaptive feedforward) controls are implemented, but no significant difference in performance is found between the two. This finding implies that, although the system under investigation could have one or more random variation factors, such as that described in (3), the processes are nevertheless stationary and the adaptive capability of the closed-loop control does not really have an opportunity to show its impact on the performance during a short-term experiment.

The present work adopts many common techniques used in a previous work on the thrust noise (Wang et al., 2005) but there are also differences. The present work focuses on the rotating drag noise, while the previous one is on the thrust noise. In both cases, the global suppression of the dominant noise is achieved. The original fan is also modified in both cases before the active control is applied. In the case of the thrust noise control, a special coincident design of $B=S=7$ is used. That modification actually represents an increase of the noise radiation from the dominant source although the increase can be minimized if smaller strut size is adopted. In the present study, however, the modifications of the inlet bellmouth and the strut size equalization serve as a significant noise reduction from the original fan with the same number of struts. In other words, the current work begins with an already quiet design version of the most popular design configuration of $B=7, S=4$. In this sense, this study goes much beyond the previous. However, a rotating antisound is more difficult to construct, and more loudspeakers are used in the current study than in the previous. For a general case where both drag and thrust noises are present at the leading radiation modes, perhaps at different frequencies for each component, a minimum of three loudspeakers would be needed to construct the antisound for the three force components.

\section{ACKNOWLEDGMENTS}

The first author thanks the Hong Kong Polytechnic University for the Ph.D. research studentship during the early stages of the work reported here. The main support came from a University grant (G-U076) and the Research Grants Council of the Government of HKSAR (Grant No. PolyU $1 / 02 C)$. Earlier work was also partially funded by the Intel Corporation.

Dowling, A. P. (1998). "Steady-state radiation from sources," in Handbook of Acoustics, edited by M. J. Crocker, (Wiley, New York), Chap. 8.

Gee, K. L., and Sommerfeldt, S. D. (2004). "Application of theoretical modeling to multichannel active control of cooling fan noise," J. Acoust. Soc. Am. 115, 228-236.

Gerard, A., Berry, A., and Masson, P. (2005). "Control of tonal noise from subsonic axial fan. Part 2: active control simulations and experiments in free field," J. Sound Vib. 288, 1077-1104.

Gerhold, C. H. (1997). "Active control of fan-generated tone noise," AIAA J. 35, 17-22.

Huang, L. (2003). "Characterizing computer cooling fan noise," J. Acoust. Soc. Am. 114, 3189-3199.

Huang, L., and Wang, J. (2005). "Acoustic analysis of a computer cooling fan,” J. Acoust. Soc. Am. 118, 2190-2200.

Lauchle, G. C., Macgillivray, J. R., and Swanson, D. C. (1997). "Active control of axial-flow fan noise," J. Acoust. Soc. Am. 101, 341-349.

Lowson, M. V. (1965). "The sound field for singularities in motion," Proc. R. Soc. London, Ser. A 286, 559-572.

Lowson, M. V. (1970). "Theoretical analysis of compressor noise," J. Acoust. Soc. Am. 47, 371-385.

Neuhaus, L., Schulz, J., Neise, W., and Moser, M. (2003). "Active control of the aerodynamic performance and tonal noise of axial turbomachines," Proc. Inst. Mech. Eng., Part A 217, 375-383.

Quinlan, D. A. (1992). "Application of active control to axial flow fans," J. Audio Eng. Soc. 39, 95-101.

Rao, N. M., Feng, J. E., Burdisso, R. A., and Ng, W. F. (2001). "Experimental demonstration of active flow control to reduce unsteady stator-rotor interaction," AIAA J. 39, 458-464.

Simonich, J., Lavrich, P., Sofrin, T., and Topol, D. (1993). "Active aerodynamic control of wake-airfoil interaction noise-experiment," AIAA J. 31, 1761-1768.

Thomas, R. H., Burdisso, R. A., Fuller, C. R., and O'Brien, W. F. (1993). "Preliminary experiments on active control of fan noise from a turbofan engine," J. Sound Vib. 161(3), 532-537.

Thomas, R. H., Burdisso, R. A., Fuller, C. R., and O’Brien, W. F. (1994). "Active control of fan noise from a turbofan engine," AIAA J. 32, 23-30.

Tyler, J. M., and Sofrin, T. G. (1962). "Axial flow compressor noise studies," SAE Trans. 70, 309-332.

Wang, J., Huang, L., and Cheng, L. (2005). "A study of active tonal noise control for a small axial flow fan," J. Acoust. Soc. Am. 117, 734-743. 